\title{
Construction of the Adiabatic Potential of a Symmetric Molecule in the Vicinity of Charged Semiconductor Surface
}

\author{
S.A. Bercha ${ }^{a, b}$, K.E. Glukhov ${ }^{a}$, L.Yu. Kharkhalis ${ }^{a}$ And M. Sznajder ${ }^{c, *}$ \\ ${ }^{a}$ Institute of Physics and Chemistry of Solid State, Uzhgorod National University, \\ 54 Voloshin St., 88000 Uzhgorod, Ukraine \\ ${ }^{b}$ Charles University, Faculty of Mathematics and Physics, Department of Surface and Plasma Science, \\ V Holesovickach 2, Prague, 18000, Czech Republic \\ ${ }^{c}$ Faculty of Mathematics and Natural Sciences, University of Rzeszów, S. Pigonia 1, 35-959 Rzeszów, Poland \\ We present a description of the symmetry-based method for the construction of the adiabatic potential of a \\ symmetric molecule near the charged semiconductor surface. For this purpose, a transformation of the adiabatic \\ potential of a free high symmetric molecule $\left(D_{3 d}\right)$ in the presence of uniform electric field is investigated. The ob- \\ tained adiabatic potential is analyzed with respect to the stability of molecule in the vicinity of charged surface, \\ as a dependence of its orientation in the electric field.
}

DOI: 10.12693/APhysPolA.129.A-120

PACS: 71.20.-b, 71.70.Ej, 61.50.Ah, 02.20.-a

\section{Introduction}

A correct solution of the issue concerning a construction of the adiabatic potential by means of group theory allows one to use the adiabatic potentials for a qualitative explanation of wide variety of physical phenomena related with vibronic coupling. In particular, these potentials are extremely useful for interpretations of IR-, Raman-, photo- and X-ray spectroscopy data, as well as in the theory of chemical reactions and catalysis. The application of adiabatic potentials can be important in the theory of phase transitions caused by the vibronic interaction (the so-called cooperative Jahn-Teller effect), too $[1,2]$.

Recently, we have shown how to construct $[3,4]$ the vibronic potential and the corresponding adiabatic potential for a molecule with $C_{3 v}$ and $D_{3 d}$ symmetries by means of the group-theory methods supplied with the Pikus method of invariants [5]. The symmetry of the molecule's normal vibrations which are active in the Jahn-Teller effect has been established there. The vibronic coupling of the $E_{\mathrm{g}}-E_{\mathrm{g}}$ type that enables one to obtain the vibronic potential energy in a matrix form as well as the adiabatic potentials, have been investigated there $[3,4]$. Studies on the interaction between a molecule and the atomic surface require taking into account that a newly prepared surface of a material is charged. Elimination of this charge from the surface involves usually a passivation procedure. This procedure relies on a surface irradiation by the hydrogen ions. On the other hand, in order to clean a surface, causing at the same time that it becomes charged, one uses a stream of flowing $\mathrm{Ar}$ atoms. Charged surface exhibits an electric field which is perpendicular to the surface.

*corresponding author; e-mail: sznajder@ur.edu.pl
It is a common practice to investigate the interaction of molecules with crystal surface in complex photoemission experiments with a parallel modeling of the energy states and density of states of both a free molecule and a molecule together with surface fragment $[6,7]$. However, the group theory methods, as it will be shown below, indicate an ambiguity of such approach. If a stream of molecules, of a given fixed orientation of incidence swoops to such a surface, there arises a problem of theoretical study on the stability of a molecule in the electric field. In order to solve this problem, we will construct below the adiabatic potential which includes the external electric field. Our study allows to predict the behavior of a free molecule in the electric field.

\section{Model of a molecule in the uniform electric field}

We will demonstrate below that the influence of an external field on a centrosymmetric molecule, oscillating near a charged surface can be described by the methods of the theory of symmetry. Our model is an extension of study presented in [3], where a molecule with $D_{3 d}$ point symmetry has been investigated in its free state. It follows from [3] that by applying the Pikus method of invariants [5] one can obtain a matrix of the vibronic interaction potential energy $D\left(Q_{1}, Q_{2}\right)$, which depends on normal vibrations $Q_{1}$ and $Q_{2}$. These vibrations, in turn, are the basic functions of the representation $E_{\mathrm{g}}$ of the $D_{3 d}$ symmetry group [3]:

$$
\begin{aligned}
& D\left(Q_{1}, Q_{2}\right)=\frac{1}{2} \omega^{2}\left(Q_{1}^{2}+Q_{2}^{2}\right) \sigma_{1}+V Q_{1} \sigma_{x} \\
& +V Q_{2} \sigma_{z}+2 W Q_{1} Q_{2}+W\left(Q_{1}^{2}-Q_{2}^{2}\right) \sigma_{z},
\end{aligned}
$$

where $V$ and $W$ are coefficients near linear and quadratic terms of the potential energy operator of the vibronic interaction, $\sigma_{1}$ denotes an unit matrix, $\sigma_{x}, \sigma_{z}$ are the corresponding Pauli matrices, and $1 / 2 \omega^{2}\left(Q_{1}^{2}+Q_{2}^{2}\right)$ is the potential energy of the molecule's normal oscillation which is described by the doubly degenerate represen- 
tation $E_{\mathrm{g}}$. The vibronic interaction is not taken into account in this potential energy. In the presence of the electric field which, in our case, is described by a vector representation of the $D_{3 d}$ symmetry group, additional terms which depend on the $\left(E_{x}, E_{y}, E_{z}\right)$ field components and their products with normal vibrations $Q_{1}$ and $Q_{2}$, should be included in Eq. (1) up to the second order. Our analysis, based on the Pikus method, shows that these additional terms take the form

$$
\begin{aligned}
& M_{1} E_{z}^{2} \sigma_{1}+M_{2}\left(E_{x}^{2}+E_{y}^{2}\right) \sigma_{1}+M_{3}\left(E_{x}^{2}-E_{y}^{2}\right) \sigma_{z} \\
& \quad+2 M_{3} E_{x} E_{y} \sigma_{x}+M_{4} E_{x} E_{z} \sigma_{z}-M_{4} E_{y} E_{z} \sigma_{x},
\end{aligned}
$$

where $M_{i}$ are arbitrary constants. Note that terms proportional to $Q_{i} E_{j}$ products vanish as ones which are incompatible with the $E_{\mathrm{g}}$ symmetry. Indeed, normal vibrations $Q_{i}$ exhibit even parity under action of the symmetry operations of the $D_{3 d}$ group, while $\boldsymbol{E}$ components possesses odd parity.

When the vibronic potential energy is presented in a matrix form $D\left(Q_{1}, Q_{2}, E_{x}, E_{y}, E_{z}\right)$, it is possible to obtain the adiabatic potential $\varepsilon_{1,2}\left(Q_{1}, Q_{2}, \boldsymbol{E}\right)$ by solving a quadratic equation that follows from the following determinant:

$$
\left|D\left(Q_{1}, Q_{2}, E_{x}, E_{y}, E_{z}\right)-\varepsilon 1\right|=0 .
$$

\section{Results and conclusions}

To analyze an influence of the electric field on the adiabatic potential, and thus, to examine a stability of a high symmetry molecule, we use a graphical representation of both solutions of Eq. (3). Cross-sections of the isoenergetic surfaces $\varepsilon_{1,2}\left(Q_{1}, Q_{2}, E_{x}, E_{y}, E_{z}\right)=$ const at arbitrary levels of the electric field $E_{z}\left(E_{x}=E_{y}=0\right)$ are presented in the left part of Fig. 1. It can be seen that with increasing values of the $E_{z}$ component $\left(E_{z} \tau\right.$ coincides with the symmetry axes of a molecule) the axial symmetry of the potential does not change. Such behavior of the first solution $\varepsilon_{1}$ is in agreement with a fact that the electric field being a vector with axial symmetry does not break the rotational symmetry of the molecule, provided that it is directed along this field.
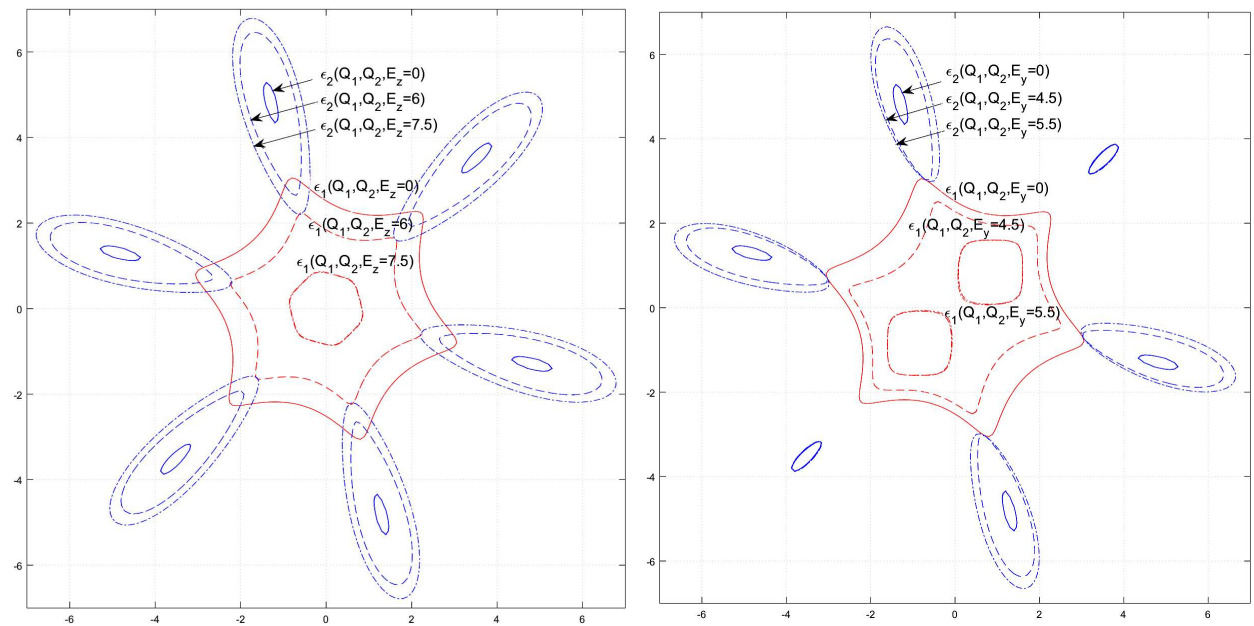

Fig. 1. Cross-sections of the adiabatic potential $\varepsilon_{1,2}\left(Q_{1}, Q_{2}, E_{x}, E_{y}, E_{z}\right)=$ const at different values of the electric field components.
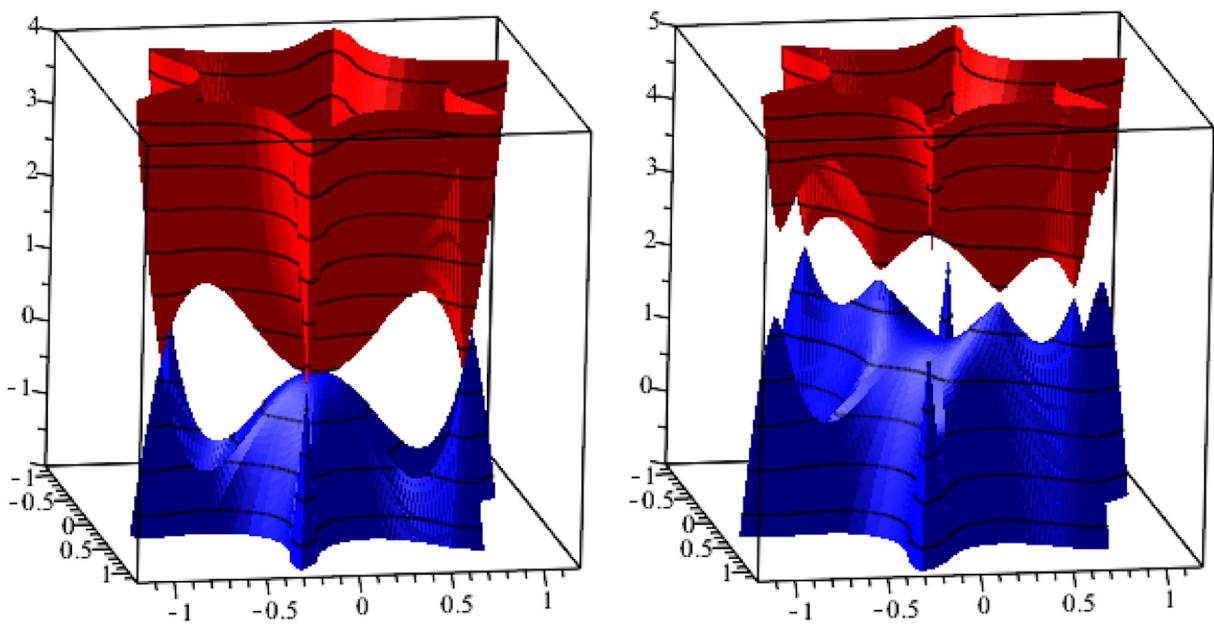

Fig. 2. Shape of the adiabatic potential $\varepsilon_{1,2}\left(Q_{1}, Q_{2}, E_{x}, E_{y}, E_{z}\right)=$ const for zero (left) and nonzero (right) electric field values. 
Let us consider the situation when a molecule enters in the field of charged surface having its symmetry axis directed parallel to this surface. In such a situation we observe different types of the symmetry lowering. In particular, in the case presented in right side in Fig. 1, when the only nonzero component of the electric field is $E_{y}$, we find that the initial configuration of the adiabatic potential with six equivalent minima is distorted in such way that all minima form equivalent pairs with different depths.

In the most general case a molecule is under an action of all nonzero electric field components. This case is compared with the zero field one in Fig. 2. As it follows from our considerations, the electric field influences drastically a shape of the adiabatic potential (all its minima become non-equivalent) and thus, this field can cause significant changes in the interatomic distances of the molecule, together with the change of its symmetry.

Thus, the above qualitative results show that at modeling of a physical system near a charged surface (e.g. by first principles methods), one should keep attention to do not preserve the symmetry constraints be imposed on the molecule's atom, since these constraints can be removed by the influence of the applied external field. The group-theoretical approach reveals the complexity of interpretations of experimental data: in the process of stability loss of the system various system symmetry configurations can occur, from the highest symmetry $D_{3 d}$ down to full asymmetric configuration.

\section{Acknowledgments}

M.S. acknowledges the support from the Centre for Innovation and Transfer of Natural Sciences and Engineering Knowledge at the University of Rzeszów. M.S. acknowledges the support from the Centre for Innovation and Transfer of Natural Sciences and Engineering Knowledge at the University of RzeszĂłw. The Authors are very grateful to Prof. D.M. Bercha for valuable discussions.

\section{References}

[1] H.A. Jahn, E. Teller, Proc. R. Soc. A Math. Phys. Sci. 161(905), 220 (1937).

[2] D.M. Bercha, S.A. Bercha, K.E. Glukhov, M. Sznajder, Acta Phys. Pol. A 126, 1143 (2014).

[3] S.A. Bercha, V.M. Rizak, UzhNU Sci. Herald (Phys.) 33, 15 (2013).

[4] S.A. Bercha, V.M. Rizak, Condens. Matter Phys. 17, 23701 (2014).

[5] G.L. Bir, G.E. Pikus, Symmetry and Strain-Induced Effects in Semiconductors, Wiley, New York 1974.

[6] N. Tsud, S.A. Bercha, R.G. Acres, M. Vorokhta, I. Khalakhan, K.C. Prince, V. Matolin, Phys. Chem. Chem. Phys. 17, 2770 (2015).

[7] N. Tsud, R.G. Acres, M. Iakhnenko, D. Mazur, K.C. Prince, V. Matolin, J. Phys. Chem. B 117, 918293 (2013). 